\title{
Preditores de piora da mobilidade ao final da internação em hospital de referência em doenças infectocontagiosas
}

\author{
Predictors of mobility impairment after hospitalization in a reference hospital among \\ individuals with infectious diseases
}

Predictores de empeoramiento de la movilidad al final de la hospitalización en un hospital de referencia para enfermedades infectocontagiosas

\author{
Ane Carolline Gonzaga Ferreira', Isabella Ribeiro Araujo², Daniella Alves Vento³, \\ Viviane Assunção Guimarães ${ }^{4}$
}

RESUMO I As doenças infectocontagiosas podem gerar complicações motoras e funcionais, prejudicando a qualidade de vida dos indivíduos acometidos. Além disso, observa-se que a própria internação hospitalar é uma causa importante de declínio funcional dos pacientes. Nesses casos, a fisioterapia visa preservar e restaurar a integralidade de órgãos e funções, promovendo a manutenção da amplitude de movimento, o ganho de força muscular, o treino de trocas posturais e de marcha, assim como a prática de exercícios aeróbios e a melhora da expansibilidade torácica de indivíduos hospitalizados. Os objetivos deste estudo foram descrever o perfil epidemiológico dos pacientes em um hospital de doenças infectocontagiosas e identificar preditores de piora motora ao final da internação. Trata-se de um estudo retrospectivo baseado na análise de prontuários de pacientes internados em 2016 que necessitaram de fisioterapia. Foram coletadas variáveis socioeconômicas e clínicas e realizada análise estatística descritiva e regressão logística binária para determinar os fatores preditores de piora da mobilidade ao final da internação. Foram avaliados 638 prontuários eletrônicos de pacientes internados de janeiro a dezembro de 2016 que necessitaram de fisioterapia, com prevalência do sexo masculino $(66,6 \%)$ e mediana de idade de 42 anos; 50,8\% dos pacientes eram portadores do vírus da imunodeficiência humana (HIV). Foram encontrados cinco fatores de risco para piora da mobilidade ao final da internação: idade, número de internações prévias, uso de ventilação mecânica, ser portador do HIV e presença de doenças oportunistas. Diante dos achados, observase a importância da atuação fisioterapêutica voltada para a melhora funcional em pacientes que cursam com internação hospitalar.

Descritores | Fatores de Risco; Limitação da Mobilidade; Hospitalização; Doenças Infecciosas.

\begin{abstract}
I Infectious diseases may lead to motor and functional complications, impairing the quality of life of affected individuals. Moreover, hospitalization itself is considered an important cause of functional decline in patients. In these cases, physiotherapy aims to preserve and restore the integrity of organs and functions, promoting the maintenance of range of motion, the gain of muscle strength, the training of postural changes and gait, as well as the practice of aerobic exercises and the improvement of thoracic expandability in hospitalized individuals. This study aimed to describe the epidemiological profile of patients at a reference hospital for infectious diseases and identify predictors of mobility impairment after hospitalization This is a retrospective study based on the
\end{abstract}

Estudo realizado no Hospital Estadual de Doenças Tropicais Dr. Anuar Auad, Goiânia, GO, Brasil.

'Hospital de Doenças Tropicais Dr. Anuar Auad (HDT) - Goiânia (GO), Brasil. E-mail: anecarolline@gmail.com. Orcid: 0000-0002-9660-9309 ${ }^{2}$ Hospital de Doenças Tropicais Dr. Anuar Auad (HDT) - Goiânia (GO), Brasil. E-mail: isabella.fisio@hotmail.com. Orcid: 0000-0002-4471-844X

3Universidade Estadual de Goiás (UEG) - Goiânia (GO), Brasil. E-mail: daniellaavento@hotmail.com. Orcid: 0000-0002-7705-1258 ${ }^{4}$ Hospital das Clínicas da Universidade Federal de Goiás (HC-UFG) - Goiânia (GO), Brasil. E-mail: vivasgui@hotmail.com. Orcid: 0000-0002-3068-8278 
analysis of medical records of patients hospitalized in 2016 who required physiotherapy. Data consisted of socioeconomic and clinical variables, and the predictors of mobility impairment after hospitalization were determined by descriptive statistics and binary logistic regression. Among the 638 electronic medical records of patients hospitalized from January to December 2016 who required physiotherapy, $66.6 \%$ were men with median age of 42 years and $50.8 \%$ were HIV-positive. We verified five risk factors for mobility impairment after hospitalization: age, number of previous hospitalizations, use of mechanical ventilation, HIV-infected patients, and presence of opportunistic infections. Our findings point to the importance of physiotherapy for improving functional mobility in hospitalized patients.

Keywords I Risk Factors; Mobility Limitation; Hospitalization; Infectious Diseases.

RESUMEN I Las enfermedades infectocontagiosas pueden ocasionar complicaciones motoras y funcionales, perjudicando la calidad de vida de las personas afectadas. Además, se observa que la propia hospitalización es una causa importante del deterioro funcional de los pacientes. En estos casos, la fisioterapia tiene como objetivo preservar y restaurar la integralidad de órganos y funciones, promoviendo el mantenimiento de la amplitud de movimiento, la ganancia de fuerza muscular, el entrenamiento de cambios posturales y de marcha, así como la práctica de ejercicios aeróbicos y la mejora de expansión torácica de los pacientes hospitalizados. Los objetivos de este estudio fueron describir el perfil epidemiológico de los pacientes en un hospital para enfermedades infectocontagiosas, así como identificar los predictores de empeoramiento motor al final de la hospitalización. Este es un estudio retrospectivo con base en el análisis de historias clínicas de pacientes hospitalizados en 2016 que requirieron fisioterapia. Se recogieron variables socioeconómicas y clínicas y se realizó análisis estadístico descriptivo y regresión logística binaria para determinar los factores predictores de empeoramiento de la movilidad al final de la hospitalización. Se evaluaron 638 historias clínicas electrónicas de pacientes hospitalizados en el período de enero a diciembre de 2016 que requirieron fisioterapia, con prevalencia de varones (66,6\%) y una mediana de edad de 42 años; el 50,8\% de los pacientes tenían el virus de la inmunodeficiencia humana (VIH). Se encontraron cinco factores de riesgo de empeoramiento de la movilidad al final de la hospitalización: edad, número de hospitalizaciones previas, uso de ventilación mecánica, ser VIH positivo y presencia de enfermedades oportunistas. Los hallazgos evidencian la importancia de la fisioterapia dirigida a la mejora funcional en los pacientes hospitalizados.

Palabras clave | Factores de Riesgo; Limitación de la Movilidad; Hospitalización; Enfermedades Infecciosas.

\section{INTRODUÇÃO}

O Brasil tem passado por uma transição epidemiológica na saúde, uma vez que a expectativa de vida da população está aumentando e as principais causas de óbito e comorbidades têm sido as doenças crônicas não-transmissíveis ${ }^{1}$.

Ao traçar um comparativo com a década de 1930, notamos que nessa época as doenças infectocontagiosas eram responsáveis por $46 \%$ do total de óbitos na população, ao passo que, em 1985, representaram apenas 7\%. Em contrapartida, as doenças cardiovasculares, neoplasias e causas externas de morte triplicaram ao longo desse período ${ }^{1,2}$.

Apesar do declínio da mortalidade resultante de doenças infecciosas e parasitárias, elas não devem ser ignoradas ou negligenciadas, pois ainda geram grande quantidade de óbitos, além de sequelas e incapacidades ${ }^{3}$. Os casos de síndrome da imunodeficiência adquirida (AIDS) representam mais de doze mil mortes por ano no Brasil desde 20094.

Existem diversas doenças infecciosas e parasitárias de interesse para a saúde pública. De forma geral, essas doenças podem afetar três sistemas orgânicos importantes: respiratório, gastrointestinal e nervoso, trazendo complicações funcionais e prejudicando a qualidade de vida dos indivíduos acometidos ${ }^{5}$.

Não são somente as doenças infectocontagiosas que podem ser acompanhadas por condições incapacitantes durante as hospitalizações. Além disso, pneumonias e exacerbações de doenças crônicas também são exemplos de enfermidades que podem resultar na perda funcional ${ }^{6}$. Menezes, Oliveira e Menezes ${ }^{7}$ relataram que a internação, e não a doença em si, podem ser a principal causa de declínio funcional em pacientes idosos; pois observa-se que, independentemente da causa de internação, o paciente pode ficar debilitado, o que reduz seu nível de mobilidade . $^{7}$.

A fisioterapia contribui para o bem-estar geral por meio de ações preventivas e reabilitadoras em pacientes no âmbito hospitalar e ambulatorial. O tratamento fisioterapêutico visa preservar e restaurar a integralidade de órgãos e funções, promovendo a manutenção da amplitude de movimento, o ganho de força muscular, o treino de 
trocas posturais e de marcha, além da prática de exercícios aeróbios e da melhora da expansibilidade torácica ${ }^{8}$.

É necessário ressaltar a importância do tratamento multiprofissional nessa população, pois o estabelecimento do vínculo entre o paciente e a equipe de saúde pode possibilitar um melhor entendimento da própria doença e seus malefícios e levá-lo para a adesão ao tratamento, o que pode resultar em menos complicações clínicas e funcionais ${ }^{5}$.

A escassez de pesquisas que identifiquem os fatores de risco para a piora da mobilidade em pacientes internados, principalmente em hospitais de doenças infecciosas, foi o que determinou o interesse por essa investigação. Desta forma, o presente estudo teve os objetivos de descrever o perfil epidemiológico dos pacientes atendidos pelo serviço de fisioterapia em um hospital de referência em doenças infectocontagiosas para avaliar o nível de mobilidade e analisar os fatores preditores de piora motora ao final da internação.

\section{METODOLOGIA}

Trata-se de um estudo retrospectivo, em que a coleta de dados ocorreu por meio da revisão dos prontuários eletrônicos dos pacientes. Para essa pesquisa, houve a dispensa do Termo de Consentimento Livre e Esclarecido.

Foram incluídos na pesquisa os pacientes adultos com idade acima de 18 anos admitidos no Hospital de Doenças Tropicais Dr. Anuar Auad (HDT) de Goiânia de janeiro a dezembro de 2016 que tiveram acompanhamento fisioterapêutico; não necessitaram de acompanhamento os pacientes que já apresentavam capacidade motora máxima - deambulação independente - durante as avaliações e reavaliações. Foram excluídos do levantamento os pacientes que faleceram antes do início do atendimento fisioterapêutico.

As seguintes variáveis socioeconômicas foram coletadas: idade, sexo, moradia, escolaridade e ocupação. E como as variáveis clínicas foram incluídas: tempo de internação, número de internações prévias, desfecho da internação, nível de mobilidade na admissão e no desfecho da internação, tipo de ventilação (espontânea ou mecânica) na admissão e na alta, uso de ventilação mecânica (VM) (quantificada em dia), se o paciente era portador do vírus da imunodeficiência humana (HIV), internação por doença infectocontagiosa, presença de doenças oportunistas e complicações hospitalares.

Para a avaliação do nível de mobilidade, o setor de fisioterapia do hospital utiliza na rotina a escala validada por Callen et al. ${ }^{9}$ Ela varia em cinco níveis de mobilidade (1 a 5) e três níveis de dependência para cada um deles (A, B e C) (Quadro 1). Esta ferramenta classifica a mobilidade como: (1) cama; (2) cama-cadeira sem descarga de peso; (3) camacadeira com descarga parcial de peso; (4) deambulação com auxílio; e (5) deambulação sem assistência. Os pacientes com nível de mobilidade de 1 a 3 não deambulam e os com nível 4 ou 5 deambulam, mesmo que com apoio 9 .

Os dados foram estruturados em banco de dados utilizando o programa Microsoft Excel 2016 e a análise estatística foi feita com o programa SPSS versão 18.0. Realizou-se a análise descritiva das características sociodemográficas e clínicas dos pacientes na internação. O teste de Shapiro-Wilk foi utilizado para verificar a normalidade das variáveis contínuas. Assim, as variáveis contínuas se apresentaram em mediana e intervalo interquartil e as categóricas em frequência e porcentagem.

Foi realizada regressão logística binária para avaliação das variáveis independentes que influenciaram na piora da mobilidade dos pacientes durante a internação. As variáveis incluídas no modelo forward $L R$ foram: idade, sexo, moradia, tempo de internação, número de internações prévias, uso de VM, dias de VM, portador do HIV, causa da internação, doenças oportunistas e complicações hospitalares. O nível de significância estatística considerado na análise foi valor de $\mathrm{p}<0,05$.

Quadro 1. Classificação do nível de mobilidade

\begin{tabular}{|c|c|c|c|c|c|}
\hline Nível de mobilidade & Cama & $\begin{array}{l}\text { Transferência leito/ } \\
\text { cadeira passiva }\end{array}$ & $\begin{array}{l}\text { Transferência leito/ } \\
\text { cadeira ativa }\end{array}$ & Deambula com auxílio & $\begin{array}{c}\text { Deambula } \\
\text { independente }\end{array}$ \\
\hline & Nível 1 & Nível 2 & Nível 3 & Nivel 4 & Nivel 5 \\
\hline$A$ & $\begin{array}{l}\text { Posicionamento e } \\
\text { mobilização passiva }\end{array}$ & $\begin{array}{l}\text { Permanece em posição } \\
\text { de poltrona no leito }\end{array}$ & $\begin{array}{l}\text { Transferência com } \\
2 \text { pessoas }\end{array}$ & $\begin{array}{l}\text { Deambula com auxílio } \\
\text { de } 2 \text { pessoas }\end{array}$ & $\begin{array}{l}\text { Independente } \\
\text { no quarto }\end{array}$ \\
\hline B & $\begin{array}{l}\text { Auxílio para mobilização } \\
\text { e posicionamento }\end{array}$ & $\begin{array}{l}\text { Transferência mecânica } \\
\text { ou com } 3 \text { pessoas }\end{array}$ & $\begin{array}{l}\text { Transferência com } \\
1 \text { pessoa }\end{array}$ & $\begin{array}{l}\text { Deambula com auxílio } \\
\text { de } 1 \text { pessoa }\end{array}$ & $\begin{array}{l}\text { Deambula fora do } \\
\text { quarto }<20 \text { metros }\end{array}$ \\
\hline$C$ & Independente no leito & $\begin{array}{l}\text { Transferência com } 2 \\
\text { pessoas }\end{array}$ & 1 pessoa supervisiona & 1 pessoa supervisiona & $\begin{array}{l}\text { Deambula fora do } \\
\text { quarto }<20 \text { metros }\end{array}$ \\
\hline
\end{tabular}




\section{RESULTADOS}

Foram analisados 638 prontuários eletrônicos de pacientes que foram internados de janeiro a dezembro de 2016 e receberam atendimento fisioterapêutico. A idade teve mediana de 42 anos e intervalo interquartil de 33 a 55 anos. As demais variáveis socioeconômicas estão descritas na Tabela 1.

\begin{tabular}{|c|c|c|}
\hline Variável & $\mathbf{N}$ & $\%$ \\
\hline $\begin{array}{l}\text { Sexo } \\
\text { Masculino } \\
\text { Feminino }\end{array}$ & $\begin{array}{r}425 \\
213\end{array}$ & $\begin{array}{l}66,6 \\
33,4\end{array}$ \\
\hline $\begin{array}{l}\text { Moradia } \\
\text { Urbana } \\
\text { Rural } \\
\text { Casa de apoio } \\
\text { Morador de rua } \\
\text { Presidiário }\end{array}$ & $\begin{array}{r}588 \\
30 \\
9 \\
7 \\
4\end{array}$ & $\begin{array}{r}92,2 \\
4,7 \\
1,4 \\
1,1 \\
0,6\end{array}$ \\
\hline $\begin{array}{l}\text { Escolaridade } \\
\text { Não informado } \\
\text { Analfabeto } \\
\text { Alfabetizado } \\
\text { Fundamental } \\
\text { Médio } \\
\text { Superior }\end{array}$ & $\begin{array}{r}237 \\
13 \\
9 \\
211 \\
135 \\
33\end{array}$ & $\begin{array}{r}37,1 \\
2,0 \\
1,4 \\
33,1 \\
21,2 \\
5,2\end{array}$ \\
\hline $\begin{array}{l}\text { Ocupação } \\
\text { Trabalhador intelectual } \\
\text { Trabalhador braçal } \\
\text { Serviços gerais } \\
\text { Agricultura } \\
\text { Aposentado } \\
\text { Do lar } \\
\text { Outros }\end{array}$ & $\begin{array}{r}17 \\
61 \\
133 \\
40 \\
78 \\
111 \\
31\end{array}$ & $\begin{array}{r}2,7 \\
9,6 \\
20,8 \\
6,3 \\
12,2 \\
17,4 \\
31,0\end{array}$ \\
\hline
\end{tabular}

Dentre os pacientes avaliados, $324(50,8 \%)$ eram portadores de HIV. Outras doenças infectocontagiosas mais prevalentes foram tuberculose (11,6\%), hanseníase $(4,2 \%)$ e hepatites virais (2\%). Além disso, encontramos as seguintes causas de internação por doenças não infectocontagiosas: acidente ofídico $(5,2 \%)$, afecções dermatológicas $(4,1 \%)$, pneumonia $(3,9 \%)$, síndrome de Guillain-Barré (2,9\%), dentre outras.

Doenças oportunistas ocorreram em 237 (37,1\%) pacientes e as mais prevalentes estão descritas na Tabela 2. Além disso, 124 (19,4\%) pacientes tiveram complicações hospitalares e as mais frequentes foram: insuficiência renal aguda $(29,8 \%)$, sepse $(23,4 \%)$, pneumonia $(18,5 \%)$ e choque séptico $(16,1 \%)$.

O tempo de internação teve mediana de 14 dias e intervalo interquartil de 8 a 24 dias e o número de internações prévias estão descritas no Gráfico 1 . Os desfechos das internações foram 441 altas $(69,1 \%), 160$ óbitos (25,1\%), 26 transferências $(4,1 \%)$ e 11 evasões hospitalares $(1,7 \%)$.
Tabela 2. Principais doenças oportunistas

\begin{tabular}{lll} 
Doenças oportunistas & $\mathbf{N}$ & $\%$ \\
\hline Toxoplasmose & 53 & 8,3 \\
Monilíase & 40 & 6,3 \\
Histoplasmose & 28 & 4,4 \\
Sífilis & 24 & 3,8 \\
Criptococose & 18 & 2,8 \\
Herpes & 18 & 2,8
\end{tabular}

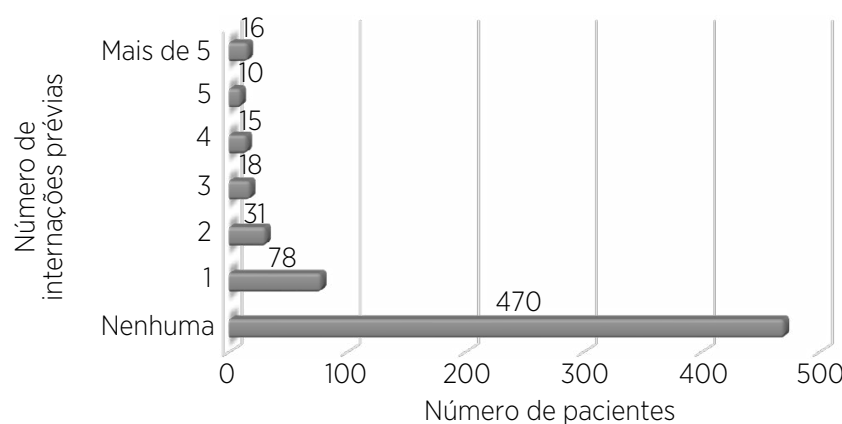

Gráfico 1. Número de internações prévias dos pacientes atendidos pela fisioterapia em 2016 no HDT/HAA

Através da avaliação respiratória, foi constatado que 226 pacientes $(35,4 \%)$ necessitaram de VM em algum momento da internação. Nestes pacientes, o tempo médio de dias em VM foi de 10,3 dias (variando de 1 a 75 dias). Quanto ao tipo de VM que utilizaram, 74,8\% dos pacientes necessitaram de ventilação invasiva, $13,7 \%$ não invasiva e $11,5 \%$ usaram tanto a invasiva quanto a não invasiva.

A avaliação do nível de mobilidade demonstrou que na admissão $392(61,4 \%)$ pacientes deambulavam e no momento do desfecho da internação 431 (67,6\%) deambulavam. Ao comparar o nível de mobilidade inicial e final, foi observado que 323 (50,6\%) pacientes melhoraram a mobilidade em pelo menos um nível de dependência, 185 (29\%) mantiveram a mesma da admissão e 130 (20,4\%) pioraram ao menos um nível de dependência.

Foi realizada regressão logística binária para avaliar as variáveis associadas com a piora da mobilidade durante a internação. $\mathrm{O}$ resultado encontrado no modelo identificou as variáveis independentes preditoras da piora da mobilidade, elencadas na Tabela 3.

Tabela 3. Preditores de piora da mobilidade na internação

\begin{tabular}{lcccr}
\multicolumn{1}{c}{ Variável } & B & Valor de p & OR & \multicolumn{1}{c}{ IC (95\%) } \\
Idade & 0,037 & $<0,001$ & 1,038 & $1,022-1,054$ \\
№ de internações prévias & 0,162 & 0,032 & 1,176 & $1,014-1,363$ \\
Uso de VM & - & $<0,001$ & 6,745 & $4,355-10,447$ \\
Portador de HIV & - & 0,043 & 1,746 & $1,018-2,993$ \\
Presença de doenças & - & 0,011 & 1,933 & $1,933-3,214$ \\
oportunistas & & & &
\end{tabular}

B: coeficiente de regressão; OR: odds ratio; IC: intervalo de confiança; VM: ventilação mecânica; HIV: virus da imunodeficiência humana. 
Observa-se que as variáveis "idade" e "uso de VM" apresentaram-se altamente preditoras de piora da mobilidade. As variáveis "idade"e "número de internações prévias" tiveram relação positiva com o desfecho; em suma, quanto maior a idade do paciente e o número de internações prévias, maior o risco de piora da mobilidade na internação. $\mathrm{O}$ uso de VM apresentou o maior odds ratio (OR); no entanto, essa variável apresentou maior variabilidade dos dados devido ao maior intervalo de confiança (IC).

\section{DISCUSSÃO}

Após análise dos 638 prontuários, observou-se maior prevalência de internação de pacientes do sexo masculino $(66,6 \%)$, oriundos de zona urbana $(92,2 \%)$, com nível de escolaridade até o ensino fundamental $(33,1 \%)$ e cujas principais ocupações foram profissionais de serviços gerais $(20,8 \%)$ e trabalhadores domésticos (17,4\%). Metade dos pacientes da amostra eram portadores do $\operatorname{HIV}(50,8 \%)$.

O estudo realizado por Gabriel, Barbosa e Vianna ${ }^{10}$ que analisou o perfil de 1.837 pacientes com HIV atendidos ambulatorialmente no município de São Paulo demonstrou maior prevalência em pacientes do sexo masculino, com escolaridade até o primeiro grau e principais ocupações eram profissionais de serviços gerais $(37,1 \%)$ e administrativos $(33,3 \%)$ ou donas de casa $(17,8 \%)$. Pode-se observar que, no Brasil, ainda há uma forte tendência desses pacientes serem de baixa renda e escolaridade ${ }^{10}$.

Em nossa amostra, 37,1\% dos pacientes tinham doenças oportunistas. Gabriel, Barbosa e Vianna ${ }^{10}$ observaram em seu estudo que $57,1 \%$ dos pacientes apresentaram doenças oportunistas, sendo as mais prevalentes: pneumocistose $(11,7 \%)$, toxoplasmose $(11,6 \%)$, candidíase $(6,2 \%)$, citomegalovirose $(3,9 \%)$, herpes $(3,5 \%)$, criptococose $(3,2 \%)$ e outras.

Ravetti e Pedroso ${ }^{11}$ em um estudo epidemiológico com 99 pacientes portadores de HIV admitidos em pronto atendimento constataram que a maior parte dos pacientes era do sexo masculino $(57,6 \%)$, apresentaram como principais desfechos da internação a alta hospitalar $(66,7 \%)$ e óbito $(12,8 \%)$ e que apenas $2,5 \%$ dos pacientes necessitaram de VM. A taxa de alta foi próxima à encontrada neste estudo (69,1\%); no entanto, a taxa de óbitos $(25,1 \%)$ e de utilização de VM $(35,4 \%)^{11}$ foram maiores nesta pesquisa.

Corroborando com nossos achados de fatores de risco para a piora da mobilidade, Ensrud et al. ${ }^{12}$ encontraram a mesma associação da idade e de internações prévias com o declínio da mobilidade em pacientes hospitalizados. Os autores apresentaram um perfil diferente do evidenciado neste estudo, pois avaliaram mulheres idosas participantes do estudo de fraturas osteoporóticas, examinando os efeitos da mobilidade e da cognição durante sua hospitalização. Foi realizada a bateria de desempenho físico curto (SPPB), que compreende testes de equilíbrio, da velocidade da marcha e da capacidade de levantar-se da cadeira. Foram obtidos resultados significantes quando os valores das baterias foram associadas à piora da mobilidade ao aumento da idade, menor cognição, histórico de internação recente e tempo de internação. Tais achados se diferiram do presente estudo em relação à influência do tempo de internação na piora da mobilidade ${ }^{12}$.

Jolley, Caldwell e Hough ${ }^{13}$ efetuaram uma pesquisa utilizando dados multicêntricos de pacientes adultos vítimas de trauma internados em unidade de terapia intensiva (UTI) sob VM, notaram que os pacientes que permaneceram na VM por um período $\geq 14$ dias apresentaram redução de amplitude de movimento e da mobilidade, devido ao imobilismo no leito. Os autores ressaltaram, ainda, que este repouso prolongado levou à redução da massa muscular e da força global, contribuindo para o prejuízo da função física relatada pelos pacientes. Esse achado pode justificar o resultado encontrado no nosso estudo em que houve relação significativa da piora da mobilidade devido ao uso de $\mathrm{VM}^{13}$.

Em contrapartida, divergindo dos nossos resultados, Jesus et al. ${ }^{14}$ avaliaram o declínio da mobilidade em pacientes clínicos e cirúrgicos, principalmente submetidos a cirurgias abdominais, internados em UTI e não encontraram relação significativa com o uso de VM, idade e motivo da internação. Os resultados achados pelos pesquisadores mostraram interação significativa da piora da mobilidade com o tempo de internação em UTI (>48 horas) e o uso de drogas vasopressoras ${ }^{14}$.

$\mathrm{O}$ estudo realizado por Richert et al. ${ }^{15}$ avaliou as alterações na função locomotora em pacientes infectados pelo HIV e os determinantes das variações no desempenho muscular do membro inferior. Assim, submeteram pacientes portadores de HIV a três testes funcionais: teste de sentar-se e levantar-se cinco vezes (SL5), teste de caminhada de seis minutos (TC6) e teste de velocidade de caminhada de dez metros. Desta forma, os resultados encontrados foram que idade, diabetes, complicações cerebrais decorrentes da infecção pelo HIV e uso de drogas injetáveis podem contribuir para a piora da função muscular dos membros inferiores. Além disso, 
ao compará-los com pessoas saudáveis da mesma idade, o desempenho desses pacientes foi pior nos testes SL5 e TC6, com desempenho deteriorado ao longo do tempo ${ }^{15}$.

A revisão feita por Christo $^{16}$ realizou uma atualização do estado epidemiológico, características clínicas e diagnóstico das complicações cognitivas no curso da infecção pelo HIV. Essa pesquisa expôs que as alterações no sistema nervoso central (SNC) em pacientes com HIV/ AIDS e as neuroinfecções podem levar a comprometimento cognitivo, emocional, motor e comportamental. Os sinais e os sintomas motores descritos são: marcha instável, fraqueza em membros, incoordenação, lentidão dos movimentos, tetraplegia espástica, dentre outros. Desta forma, é possível que com a evolução da doença e das infecções oportunistas que afetam o SNC, estes pacientes tenham tendência para piora do nível de mobilidade ${ }^{16}$.

A literatura é escassa em estudos sobre preditores de piora da mobilidade em pacientes internados, principalmente cujo perfil seja de acometimento por doenças infectocontagiosas. Portanto, considera-se importante esse tipo de estudo para que os profissionais de saúde, sobretudo fisioterapeutas, possam conhecer os fatores de risco para esse desfecho e, assim, intervir precocemente e de forma mais pontual para evitar a piora motora e funcional dos pacientes durante a internação.

As limitações apresentadas pelo estudo são referentes à dificuldade de realizar busca em fonte secundária de dados, pois nota-se a presença de registros incompletos e ausência de dados importantes. Além disso, outra limitação é quanto à heterogeneidade dos pacientes da amostra, que considerou enfermos por doenças infecciosas, acidentes por animais, doenças dermatológicas, entre outras causas. Portanto, sugerem-se novas investigações considerando grupos específicos de doenças encontradas em hospitais com o mesmo perfil do presente estudo.

\section{CONCLUSÃO}

Diante dos achados deste estudo, constatou-se que a idade, o número de internações prévias, o uso de VM, se o paciente é portador de HIV e a presença de doenças oportunistas são preditores de piora da mobilidade em pacientes internados em um hospital de doenças tropicais. É de suma importância um olhar mais atento dos fisioterapeutas para manter e/ou melhorar o nível motor e funcional dos pacientes que apresentam tais fatores de risco.

Sugere-se a realização de novas pesquisas que identifiquem os fatores responsáveis pela piora motora em pacientes hospitalizados, especialmente aqueles que são internados em hospitais de doenças infecciosas, visando uma melhor abordagem da equipe a estes pacientes.

\section{REFERÊNCIAS}

1. Pereira RA, Alves-Souza RA, Vale JS. O processo de transição epidemiológica no Brasil: Uma revisão de literatura. Rev Cient FAEMA. 2015;6(1):99-108. doi: 10.31072/rcf.v6i1.322

2. Prata PRA. Transição epidemiológica no Brasil. Cad Saude Publica. 1992;8(2):168-75. doi: 10.1590/S0102-311X1992000200008

3. Muñoz SS, Fernandes APM. As Doenças Infecciosas e Parasitárias e seus Condicionantes Ambientais [Internet]. São Paulo: Universidade de São Paulo; 2013 [cited 2020 May 10]. Available from: https://midia.atp.usp.br/plc/ju0004/impressos/ ju0004_01.pdf

4. Brasil. Ministério da Saúde. Boletim Epidemiológico: HIV-AIDS [Internet]. Brasília; 2015 [cited 2020 May 10]. Available from: http://www.aids.gov.br/pt-br/pub/2015/ boletim-epidemiologico-hivaids-2015

5. Nobre AQT, Costa IS, Bernardes KO. A fisioterapia no contexto do HIV/AIDS. Fisioter Mov [Internet]. 2008 [cited 2020 May 10];21(4):11-8. Available from: https://periodicos.pucpr.br/index. php/fisio/article/view/19193/18519

6. Zisberg A, Shadmi E, Gur-Yaish N, Tonkikh O, Sinoff G. Hospitalassociated functional decline: the role of hospitalization processes beyond individual risk factors. J Am Geriatr Soc. 2015;63:55-62. doi: 10.1111/jgs.13193

7. Menezes C, Oliveira VRC, Menezes RL. Repercussões da hospitalização na capacidade funcional de idosos. Movimenta [Internet]. 2010 [cited 2020 May 10];3(2):77-84. Available from: https://www.revista.ueg.br/index.php/movimenta/article/ view/7175

8. Bispo Júnior JP. Fisioterapia e saúde coletiva: desafios e novas responsabilidades profissionais. Cienc Saude Colet. 2010;15(1):1627-36. doi: 10.1590/S1413-81232010000700074

9. Callen BL, Mahoney JE, Wells TJ, Enole M, Hughes S. Admission and discharge mobility of frail hospitalized older adults. Medsurg Nursing [Internet]. 2004 [cited 2020 May 10];13(3):156-64. Available from: https://pubmed.ncbi.nlm.nih.gov/15219163/

10. Gabriel R, Barbosa DA, Vianna LAC. Perfil epidemiológico dos clientes HIV/AIDS da unidade ambulatorial de hospital escola de grande porte - Município de São Paulo. Rev Lat Am Enfermagem. 2005;13(4):509-13. doi: 10.1590/ S0104-11692005000400008

11. Ravetti CC, Pedroso ERP. Estudo das características epidemiológicas e clínicas de pacientes portadores do vírus da imunodeficiência humana em Pronto Atendimento do Hospital das Clínicas da Universidade Federal de Minas Gerais. Rev Soc Bras Med Trop. 2009;42(2):114-8. doi: 10.1590/S0037-86822009000200004

12. Ensrud KE, Lui L, Paudel ML, Schousboe JT, Kats AM, Cauley $J A$, et al. Effects of mobility and cognition on hospitalization and inpatient days in women in late life. J Gerontol A Biol Sci Med Sci. 2017;72(1):82-8. doi: 10.1093/gerona/glw040

13. Jolley SE, Caldwell E, Hough CL. Factors Associated with receipt of physical therapy consultation in patients requiring 
prolonged mechanical ventilation. Dimens Crit Care Nurs. 2014;33(3):160-7. doi: 10.1097/DCC.0000000000000040

14. Jesus FS, Paim DM, Brito JO, Barros IA, Nogueira TB, Martinez $B P$, et al. Declínio da mobilidade dos pacientes internados em unidade de terapia intensiva. Rev Bras Ter Intensiva. 2016;28(2):114-9. doi: 10.5935/0103-507X.20160025
15. Richert L, Brault M, Mercié P, Dauchy FA, Bruyand M, Greib $C$, et al. Decline in locomotor functions over time in HIVinfected patients. AIDS. 2014;28(10):1441-9. doi: 10.1097/ QAD.0000000000000246

16. Christo PP. Alterações cognitivas na infecção pelo HIV e AIDS. Rev Assoc Med Bras. 2010;56(2):242-7. doi: 10.1590/ S0104-42302010000200027 